\title{
Solar supergranulation revealed by granule tracking
}

\author{
M. Rieutord, N. Meunier ${ }^{\star}$, T. Roudier, S. Rondi, F. Beigbeder, and L. Parès \\ Laboratoire d'Astrophysique de Toulouse et Tarbes, UMR 5572, CNRS et Université Paul Sabatier Toulouse 3, 14 avenue E. Belin, \\ 31400 Toulouse, France \\ e-mail: [rieutord;roudier; francis.beigbeder;pares]@ast.obs-mip.fr;nmeunier@obs.ujf-grenoble.fr; \\ rondi@wanadoo.fr
}

Received 16 November 2007 / Accepted 19 December 2007

\section{ABSTRACT}

\begin{abstract}
Context. Supergranulation is a pattern of the velocity field at the surface of the Sun, which has been known about for more than fifty years, however, no satisfactory explanation of its origin has been proposed.

Aims. New observational constraints are therefore needed to guide theoretical approaches which hesitate between scenarios that either invoke a large-scale instability of the surface turbulent convection or a direct forcing by buoyancy.

Methods. Using the 14-Mpixel CALAS camera at the Pic-du-Midi observatory, we obtained a 7.5 h-long sequence of high resolution images with unprecedented field size. Tracking granules, we have determined the velocity field at the Sun's surface in great detail from a scale of $2.5 \mathrm{Mm}$ up to $250 \mathrm{Mm}$.

Results. The kinetic energy density spectrum shows that supergranulation peaks at $36 \mathrm{Mm}$ and spans on scales ranging between $20 \mathrm{Mm}$ and $75 \mathrm{Mm}$. The decrease of supergranular flows in the small scales is close to a $k^{-2}$-power law, steeper than the equipartition Kolmogorov one. The probability distribution function of the divergence field shows the signature of intermittency of the supergranulation and thus its turbulent nature.
\end{abstract}

Key words. convection - turbulence - Sun: photosphere

\section{Introduction}

Supergranulation was discovered by Hart (1954) using Doppler images of the Sun. It appeared as an essentially horizontal flow field at a typical scale of $30 \mathrm{Mm}$. The origin of this flow field was first thought to be related to the second ionization of helium, which provides some latent heat at a depth around $10 \mathrm{Mm}$, compatible with their typical size (Simon \& Weiss 1968). This scenario has been much debated because of the weakness of the effect and the apparent vigour of the supergranular flow. Other ways of generating this velocity scale rely on large-scale instabilities of the surface turbulent flow (Rieutord et al. 2000), triggered by the strong density stratification. In this scenario, kinetic energy of granules, the small-scale convective cells, is thought to be piped to a larger scale by an AKA-like effect (Gama et al. 1994). However, other kinds of large-scale instabilities are still possible, like a convective instability triggered by fixed-flux boundary conditions imposed by the small-scale granular convection (Rincon \& Rieutord 2003). In this approach, the cooling resulting from the granulation does not suppress the convective instability of the larger scales; because all the heat flux is carried by the small scale, none is carried by the large scale instability, which shows little temperature fluctuations (see Meunier et al. 2007a). Besides these scenarios, recent observations of Gizon et al. (2003) added some wavelike properties to supergranulation.

Obviously, the supergranulation theory needs guidance from every available observational constraint. This letter presents the results of the observations issued from the CALAS project (a

\footnotetext{
* Present address: Laboratoire d'Astrophysique, Observatoire de Grenoble, BP 53, 38041 Grenoble Cedex 9, France
}

CAmera for the LArge scales of the Sun; see Meunier et al. 2005), collecting a sequence of high resolution wide field images, which have allowed us to capture the evolution of a hundred supergranules at the disc centre during $7.5 \mathrm{~h}$. We can thus give new constraints on the dynamics of the solar surface in the supergranulation range. After a brief description of the data set (Sect. 2), we show the spectral side of the supergranular flow (Sect. 3) as well as the probability distribution functions of the divergences (Sect. 4); first conclusions follow.

\section{Observational techniques and data reduction}

\subsection{Data set}

On 13 March 2007, we observed the Sun at disc centre during $7.5 \mathrm{~h}$ using the Lunette Jean Rösch at Pic du Midi, a $50 \mathrm{~cm}-$ refractor. Images were taken at $\lambda=575 \pm 5 \mathrm{~nm}$, with a 14 Mpixel CMOS-camera $(4560 \times 3048$ pixels $)$, with 0.115 arcsec/pixel, thus covering of $524 \times 350 \operatorname{arcsec}^{2}$ (see Fig. 1). 10811 images were obtained with a regular cadence of one every $2.5 \mathrm{~s}$. Two independent series of $\sim 1400$ images were then extracted. They sample the same solar signal with a period of $20 \mathrm{~s}$ but are noised differently by the Earth atmospheric perturbations. The comparison between the outputs of both series allows us to evaluate the influence of the seeing and test the robustness of the results with respect to this noise.

Because of tracking difficulties, the common field of each series was reduced to $\sim 400 \times 300 \operatorname{arcsec}^{2}$, thus covering a surface of $290 \times 216 \mathrm{Mm}^{2}$ on the Sun (Fig. 1).

After recentering, subimages were $k-\omega$ filtered (with a threshold of $7 \mathrm{~km} \mathrm{~s}^{-1}$ ), so as to remove, as much as possible, 


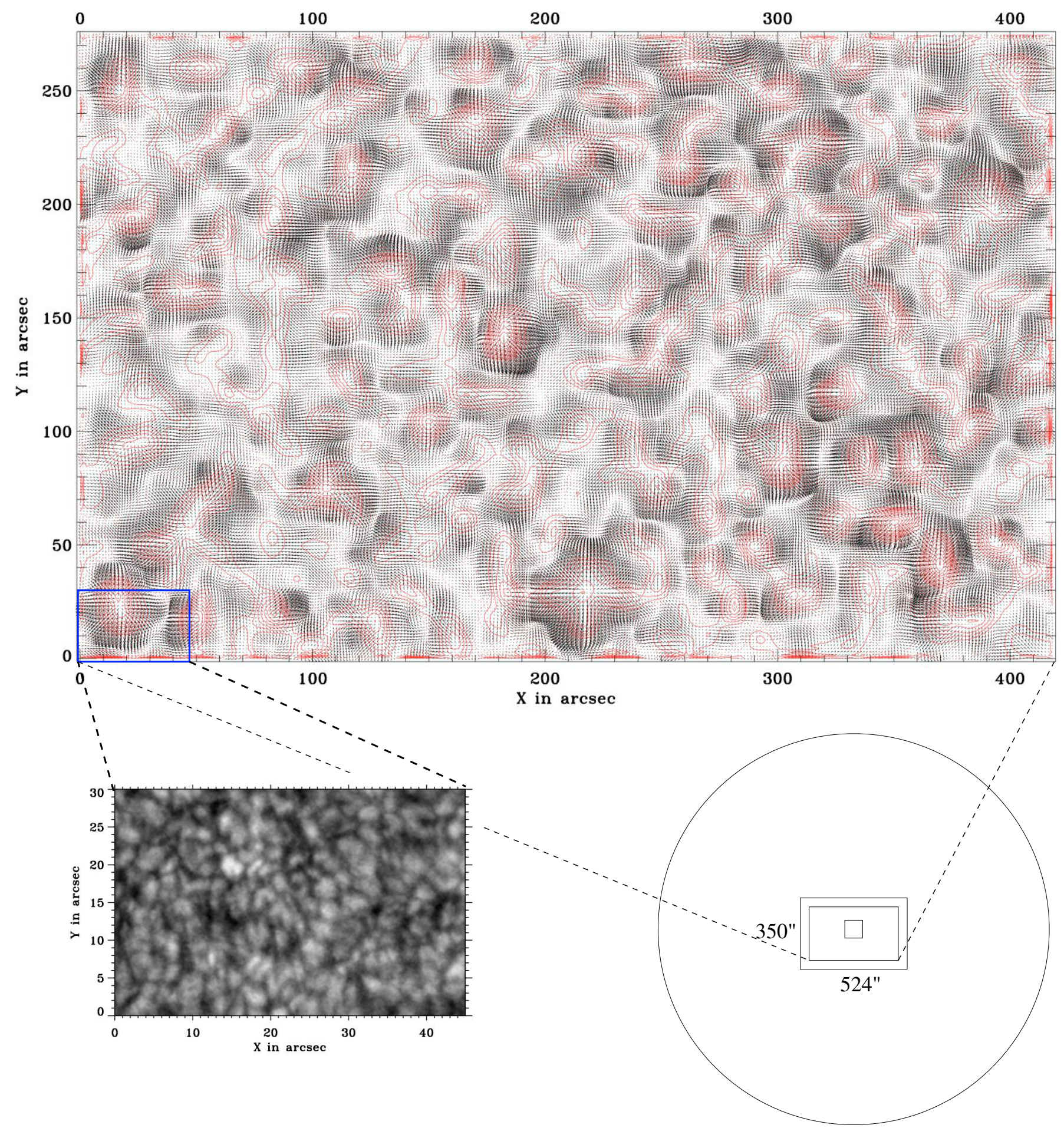

Fig. 1. Bottom right: the CALAS field of view on the Sun; the large rectangle indicates the size of the images, the smaller one shows the field where the velocities could be computed, while the small square shows the field of view of the SOT instrument on the Hinode satellite when tracking granules. Top: the supergranulation velocity field with the divergence contours superimposed (scales shorter than $8 \mathrm{Mm}$ have been filtered out). A time-window of 150 min was used. Bottom left: a zoom on granulation showing the relative sizes of granules and supergranules.

Earth atmospheric distortion, which is the main source of noise for velocity measurements (Tkaczuk et al. 2007).

\subsection{Velocity fields}

Horizontal velocity fields have been obtained using the CST granule tracking algorithm (Roudier et al. 1999; Rieutord et al. 2007). As shown in Rieutord et al. (2001), granules' motions trace large-scale velocity fields when the scale is larger than $2.5 \mathrm{Mm}$. Hence, we sampled the velocity field with a bin of 12 pixels $(\sim 1 \mathrm{Mm})$.

The velocities are obtained by tracking the granules during a given time window. Thus, we have access to average velocity components, namely $\bar{v}_{x}(i, j)$ and $\bar{v}_{y}(i, j)$, where the overline refers to the time averaging imposed by the time window. This averaging improves the signal-to-noise ratio, but naturally 


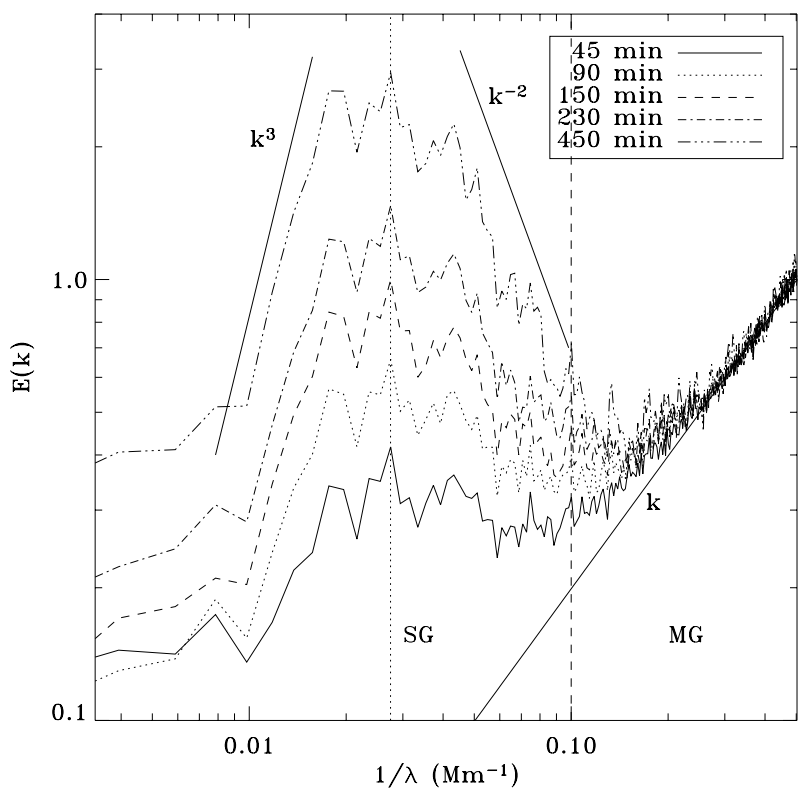

Fig. 2. Kinetic energy spectra obtained for various time windows. The vertical dotted line indicates the position of the peak at $36.4 \mathrm{Mm}$. The vertical dashed line emphasizes the $10 \mathrm{Mm}$ scale, usually taken as the upper limit of mesogranular scale. Two power laws are shown on each side of the peak, as well as the one of small-scale noise.

decreases the time resolution. Typically, the shortest time window that can be used is $30 \mathrm{~min}$.

Although the field is large, projection effects of the spherical Sun are still of weak influence. At most, in the field corners, the correction on the velocity would be less than $4 \%$, which is much less than the noise.

We show in Fig. 1 an example of these velocity fields. The small-scale components of the flow (with scales below $8 \mathrm{Mm}$ ) were filtered out using Daubechies wavelets (see Rieutord et al. 2007). Figure 1 shows that robust steady supergranules live among a wide variety of flow structures illustrating the turbulent nature of these scales and their wide spectral range.

\section{The kinetic energy spectrum}

A convenient way to view the dynamics of a flow is to examine the spectral content of the velocity field. We therefore computed the spectral density of kinetic energy $E(k)$ associated with the horizontal flows that we can measure. It is such that

$\frac{1}{2}\left\langle\bar{v}^{2}\right\rangle=\int_{0}^{\infty} E(k) \mathrm{d} k$.

$E(k)$, which is computed in the same way as in Rieutord et al. (2000), is displayed in Fig. 2 for the flows determined with various time-windows. The short time-windows of $45 \mathrm{~min}$ give us 10 independent spectra, which are averaged together, while the whole series 450 min time-window gives us only one spectrum. Note that no spatial filtering was applied to the velocity field. We see that $E(k) \propto k$ at small scale, which is the signature of decorrelated random noise.

These spectra clearly show the emergence of the spectral range of the supergranulation as the length of the timewindow increases. Let us point out the remarkable stability of the wavenumber of the spectral peak when the time averaging is changed. This demonstrates that supergranulation is a genuine velocity field at the Sun's surface. It is not the consequence

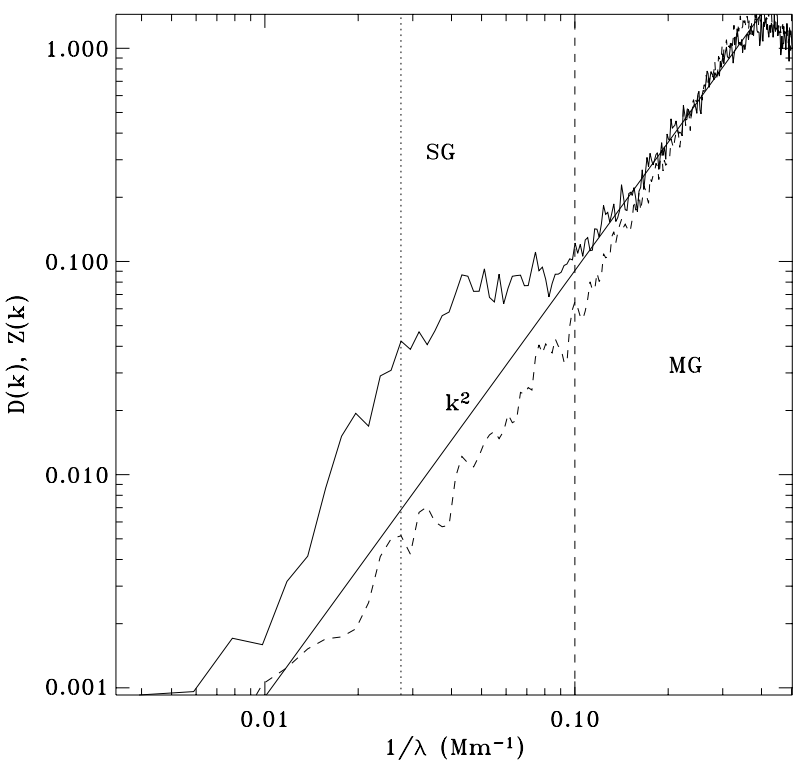

Fig. 3. Same as in Fig. 2 but for the horizontal divergence of the flow, $\partial_{x} v_{x}+\partial_{y} v_{y}$ (solid line), and for the vertical vorticity, $\partial_{x} v_{y}-\partial_{y} v_{x}$ (dashed line); the supergranulation peak is clearly visible at $\lambda=36 \mathrm{Mm}$ in the divergence spectrum but absent in the vorticity one. A $k^{2}$-power law is given for comparison.

of time-averaging the small, fast turning-over small scales. Of course, if the averaging interval is long enough (i.e. of the order of the turn-over time scale of supergranulation), the spectral peak will move to ever larger scales before disappearing. The maximum of the spectral density is at a wavelength of $36 \mathrm{Mm}$. The FWHM of the peak indicates that supergranulation occupies the range of scales of $[20,75] \mathrm{Mm}$.

The present mean value of the diameter of supergranules is slightly higher than the previous determinations. For instance, Meunier et al. (2007b) find a mean diameter of $31.4 \mathrm{Mm}$ with a technique based on the segmentation of the divergence field derived from velocities issued from a local correlation technique applied to SOHO/MDI white light images. Del Moro et al. (2004) also use a divergence field but derived from time-distance helioseismology; they find a mean size of $27 \mathrm{Mm}$, significantly smaller than ours.

Both of these results are based on the divergence field and its segmentation. The histogram of sizes is then used to determine the mean diameter of a supergranule. Such a technique necessarily underestimates the actual scale of supergranular flows as only part of it (the positive divergences) is used. Our spectra, which directly result from the measured horizontal velocities, incorporate all the components of the flow at supergranulation scale, and thus better reflect the dynamical state. Note that a blind use of the divergence spectrum in Fig. 3 would point to a scale of $\sim 23 \mathrm{Mm}$.

In Fig. 2 we also indicate the best-fit power laws, which mimic the sides of the supergranulation peak. We find that $E(k) \sim k^{3}$ on the large-scale side and $E(k) \sim k^{-2}$ on the small-scale one. This latter power law is steeper than the $-5 / 3$ Kolmogorov one and may be an effect of density stratification.

To complete the spectral picture, we also show, in Fig. 3, the spectra of the horizontal divergence of the velocity field and the vertical vorticity. Beyond the $k^{2}$-dependence of the spectral densities, which is a consequence of the derivative of uncorrelated noise, we can see that the divergence spectrum clearly shows the supergranulation peak, while the vertical vorticity shows no 


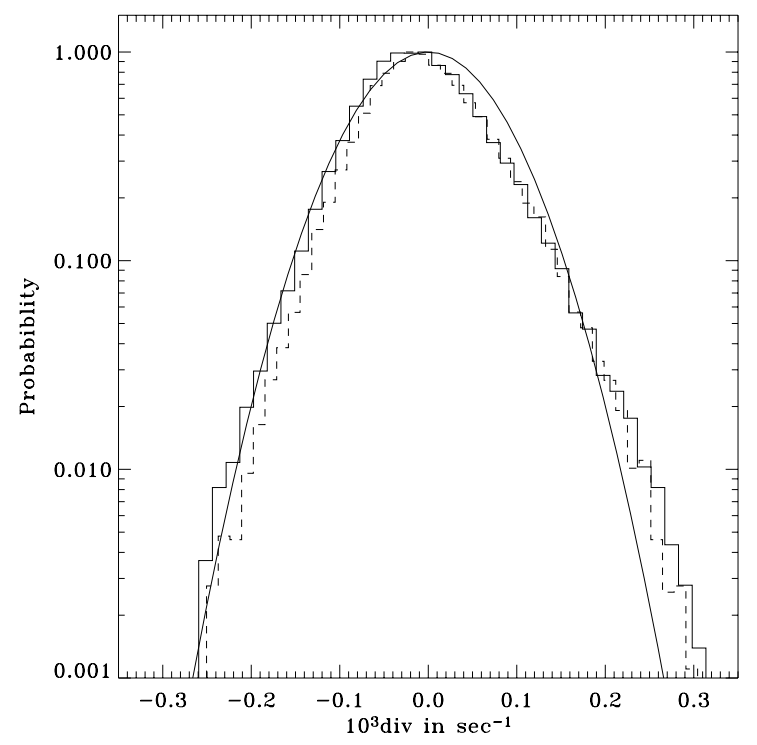

Fig. 4. Histograms of the divergences; solid and dashed lines have the same solar signal but a different noise from the Earth atmosphere. Scales below $8 \mathrm{Mm}$ have been filtered out. A Gaussian distribution with the same standard deviation is overplotted for comparison.

signal in this range. The vorticity does show some weak signal however, but in the mesogranulation range, below $10 \mathrm{Mm}$.

\section{Probability density functions}

Another way to look at the velocity fields is to consider the probability density functions. Such distributions have been computed for the velocity field, its divergence and its curl. We show in Fig. 4 the distribution of the divergence field when scales shorter than $8 \mathrm{Mm}$ have been filtered out of the velocity field. This distribution clearly shows a positive wing larger than the Gaussian one, associated with a skewness of 0.38 . We also note that the noise of the Earth atmosphere is weak enough, and does not perturb the result. This trend to an exponential distribution is a signature of intermittency (e.g. Frisch 1995). This result, with the same asymmetry between positive and negative values, was also observed by Meunier et al. (2007b) with a completely different set of data and method. Thus, the intermittency of supergranulation seems to be a robust property. Exponential wings are usually less visible on the velocity (e.g. Vincent \& Meneguzzi 1991), and, indeed, are barely noticeable in our data. As far as vorticity is concerned, the noise is unfortunately too high to give convincing measurements.

\section{Conclusions}

For the first time, it has been possible to follow the motion of well-resolved granules in a large field of view. The spectral peak of supergranulation has thus been determined in a very direct manner. According to our data, supergranulation has the most energetic motions at a scale of $36 \mathrm{Mm}$ and encompasses all the scales ranging from 20 to $75 \mathrm{Mm}$, as shown by the FWHM of the peak. Except for its amplitude, this peak is not sensitive to the time window used to measure the granules' motions. The signal also clearly appears in the divergence spectral density, but not in the vorticity; it is likely that vorticity at supergranulation scale near the Sun's equator is much weaker and does not emerge from the noise.

Finally, our data confirm the fact that supergranulation has a noticeable degree of intermittency, clearly appearing in the distribution of positive divergence values.

As far as the origin of supergranulation is concerned, the scenarios mentioned in the introduction can be tested with these data, either in the real space with velocity fields like the one shown in Fig. 1 or in the spectral space with the given spectra.

Further work on the observational side will focus on the determination of the third component of the velocity field, the increase of the field size and the reduction of the noise, so as to further constrain the dynamics of scales from the granulation one to the $100 \mathrm{Mm}$ one.

Acknowledgements. The CALAS project has been financially supported by the French ministery of education (ACI), by the Programme National Soleil-Terre of CNRS and the Observatoire Midi-Pyrénées. We are also very grateful to the "Groupe d'Intrumentation des Grands Télescopes (GIGT)" of the laboratory, for their technical help at various phase of the project, especially to Sébastien Baratchart and Elodie Bourrec. We also wish to thank René Dorignac for his efficient support in mechanical realizations and Philippe Saby for his help in sorting out the right computing hardware. S.R. wishes to thank the CNRS for its support during his $\mathrm{Ph} . \mathrm{D}$. Thesis which much contributed to the project.

\section{References}

Del Moro, D., Berilli, F., Duvall, T., \& Kosovichev, A. G. 2004, Sol. Phys., 221, 23

Frisch, U. 1995, Turbulence: the legacy of A. N. Kolmogorov (Cambridge University Press)

Gama, S., Vergassola, M., \& Frisch, U. 1994, J. Fluid Mech., 260, 95

Gizon, L., Duvall, T. L., \& Schou, J. 2003, Nature, 421, 43

Hart, A. B. 1954, MNRAS, 114, 17

Meunier, N., Rondi, S., Tkaczuk, R., Rieutord, M., \& Beigbeder, F. 2005, in Large-scale Structures and their Role in Solar Activity, ed. K. Sankarasubramanian, M. Penn, \& A. Pevtsov, ASP Conf. Ser., 346, 53 Meunier, N., Tkaczuk, R., \& Roudier, T. 2007a, A\&A, 463, 745

Meunier, N., Tkaczuk, R., Roudier, T., \& Rieutord, M. 2007b, A\&A, 461, 1141 Rieutord, M., Roudier, T., Malherbe, J. M., \& Rincon, F. 2000, A\&A, 357, 1063 Rieutord, M., Roudier, T., Ludwig, H.-G., Nordlund, Å., \& Stein, R. 2001, A\&A, 377, L14

Rieutord, M., Roudier, T., Roques, S., \& Ducottet, C. 2007, A\&A, 471, 687

Rincon, F., \& Rieutord, M. 2003, in SF2A-2003: Semaine de l'Astrophysique Francaise, ed. F. Combes, D. Barret, T. Contini, \& L. Pagani, 103

Roudier, T., Rieutord, M., Malherbe, J., \& Vigneau, J. 1999, A\&A, 349, 301

Simon, G. W., \& Weiss, N. O. 1968, Z. Astrophys., 69, 435

Tkaczuk, R., Rieutord, M., Meunier, N., \& Roudier, T. 2007, A\&A, 471, 695

Vincent, A., \& Meneguzzi, M. 1991, J. Fluid Mech., 225, 1 